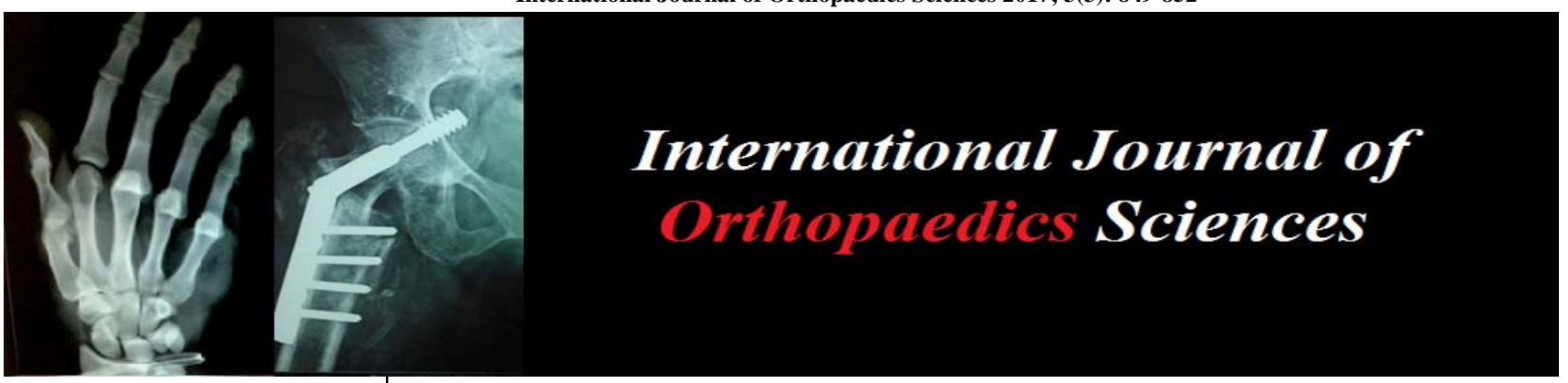

ISSN: $2395-1958$

IJOS 2017; 3(3): 849-852

(C) 2017 IJOS

www.orthopaper.com

Received: 26-05-2017

Accepted: 27-06-2017

Dr. Nirmal D Patil

MS(Ortho), SMO Nair Hospital,

Mumbai, Maharashtra, India

Dr. Hiren Patel

MS(Ortho), SMO Nair Hospital,

Mumbai, Maharashtra, India

Dr. Jayesh V Baviskar

MS(Ortho), Assistant Prof Nair

Hospital, Mumbai, Maharashtra,

India

Dr. Naushad Hussain

MS (Ortho), Prof and Head of

Unit, Nair Hospital, Mumbai,

Maharashtra, India
Correspondence

Dr. Nirmal D Patil

MS(Ortho), SMO Nair Hospital,

Mumbai, Maharashtra, India

\section{Chronic haematogenous osteomyelitis of fibula diaphysis with pathological fracture and review of literature}

\author{
Dr. Nirmal D Patil, Dr. Hiren Patel, Dr. Jayesh V Baviskar and \\ Dr. Naushad Hussain
}

DOI: https://doi.org/10.22271/ortho.2017.v3.i31.126

\section{Abstract}

Introduction: Management of fibular osteomyelitis presents various challenges with respect to stability of ankle mortise, poor quality of periosteum and fibular pseudoarthrosis.

Case History: We present a case of a 6 year male child with chronic osteomyelitis of fibula and pathological fracture, who was operated with resection of fibula with proximal and distal tibio-fibular syndesmotic fixation. Patient was followed up for One year and was noted to have fibular pseudoarthrosis and $5^{\circ}$ valgus deformity of ankle.

Discussion: Progressive valgus deformity at the ankle is a known complication that can occur many years after index fibular resection. We noticed a ankle valgus deformity at 3 month post-op which was non-progressive till 1 year follow-up. The valgus was traced retrospectively to be due to syndesmotic fixation of the fibular remnant after fibular resection, thereby adding a valgus force to ankle mortice. This could have been avoided by inserting the syndesmotic screw before fibular resection. This cause should also be kept in mind while evaluating such patients. These patients should also be followed up to detect fibular pseudoarthrosis and progressive valgus ankle deformity.

Keywords: Fibula, Pseudarthrosis, Valgus deformity, Ankle, Children, Osteomyelitis

\section{Introduction}

Osteomyelitis of the fibula is uncommon. The treatment of choice for chronic osteomyelitis is sequestrectomy initially with diaphyseal resection of the fibula reserved for recurrence ${ }^{[1]}$. Many authors have described progressive valgus deformity of the ankle following iatrogenic fibula pseudoarthrosis in children ${ }^{[2,3,4]}$. Lagenskiold ${ }^{[5]}$ advocated distal tibiofibular synostosis to stabilize the ankle and prevent progressive valgus deformity. Goh et al ${ }^{[6]}$ suggested the distal fibular function can be restored following fixing the fibular remnant to tibia. This strategy however may not prevent ankle valgus and hence close follow-up till skeletal maturity is advised. However there are discrepancies in literature regarding of occurrence of valgus deformity following Lagenskiold operation [Soo et al, Moon et al ${ }^{[7,8]}$ vs Fragniere et al ${ }^{[9]}$ ].

\section{Case history}

A 6 year old school going male child presents with pain, inability to bear weight with a history of discharging wound over the lateral aspect of distal leg following a history of fall one month ago. He had a history of fever one year ago with occurrence of a boil over the lateral aspect of distal leg. Incision and drainage was done and he was kept on antibiotics in another institute (details of organism and antibiotics are unknown). The symptoms resolved and the patient was pain free until a month ago. On examination there was a puckered wound adherent to the bone over the distal $1 / 3^{\text {rd }}$ fibula with a sinus discharging yellowish purulent material (Fig 1).

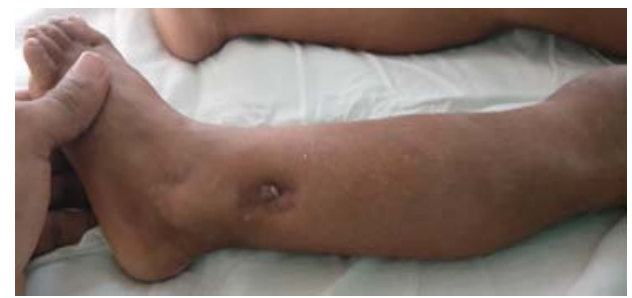

Fig 1: Sinus with yellowish purulent discharge at the distal $1 / 3^{\text {rd }}$ of lateral aspect of left leg $\sim 849 \sim$ 
Tenderness was elicited at the distal fibula. The pain score was 6 using Wong Baker faces pain rating scale. Active ankle Range was $10^{\circ}$ Dorsiflexion to $10^{\circ}$ plantar flexion which was painful. Patient was evaluated with an X-ray and MRI which confirmed the diagnosis of osteomyelitis of fibula with a pathological fracture (Fig 2 and Fig 3).

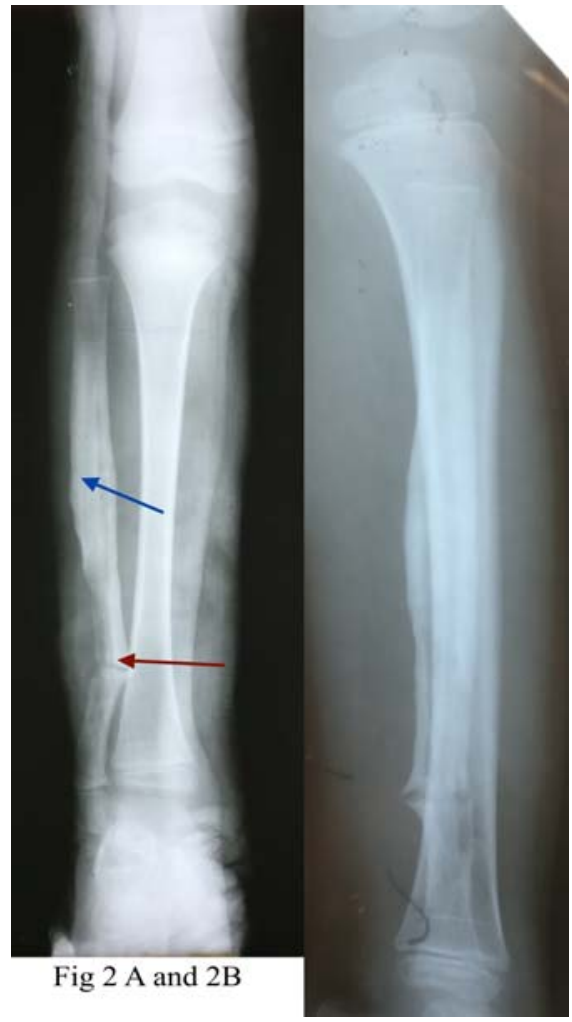

Fig 2: Preoperative Antero-posterior and Lateral $\mathrm{X}$ ray showing involucrum and sequestrum (blue arrow) and pathological fracture with poor periosteal reaction (red arrow).

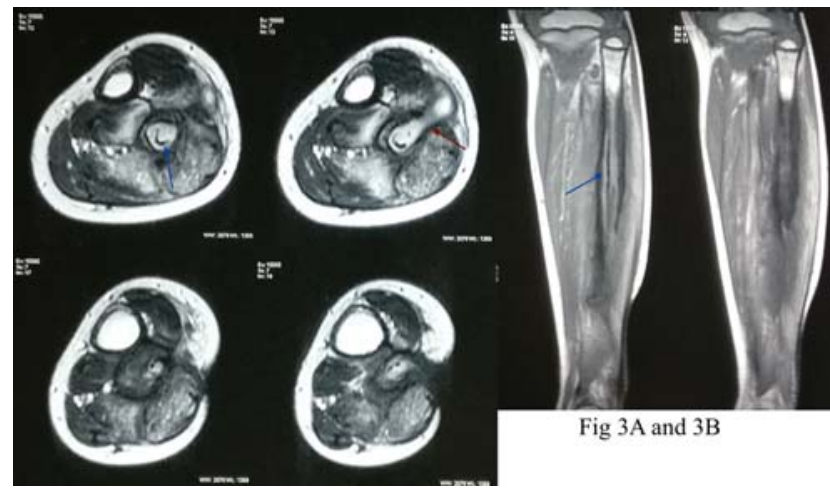

Fig 3: Axial and coronal MRI images with sequestrum (Blue arrow) and sinus tract and cloacae (Red arrow)

The WBC counts were 14,000 cells/ $\mu$ l (Normal $=4000$ $11000 / \mu \mathrm{l}) \mathrm{ESR}$ was $40 \mathrm{~mm}$ at end of 1 hour (Normal $=20$ $\mathrm{mm}$ ) and CRP was $0.85 \mathrm{mg} / \mathrm{dl}$ (normal= $0-1 \mathrm{mg} / \mathrm{dl}$ ). Culture report of the discharge revealed Methicillin resistant Staphylococcus aureus sensitive to Linezolid. The patient was planned for sequestrectomy SOS sub periosteal resection with distal tibio-fibular syndesmotic fixation.

A lateral skin incision on the fibula was taken with excision of the sinus. The periosteum was incised and sub periosteal exposure was done. The periosteum at the site of pathological fracture was noted to be damaged (Fig 4).

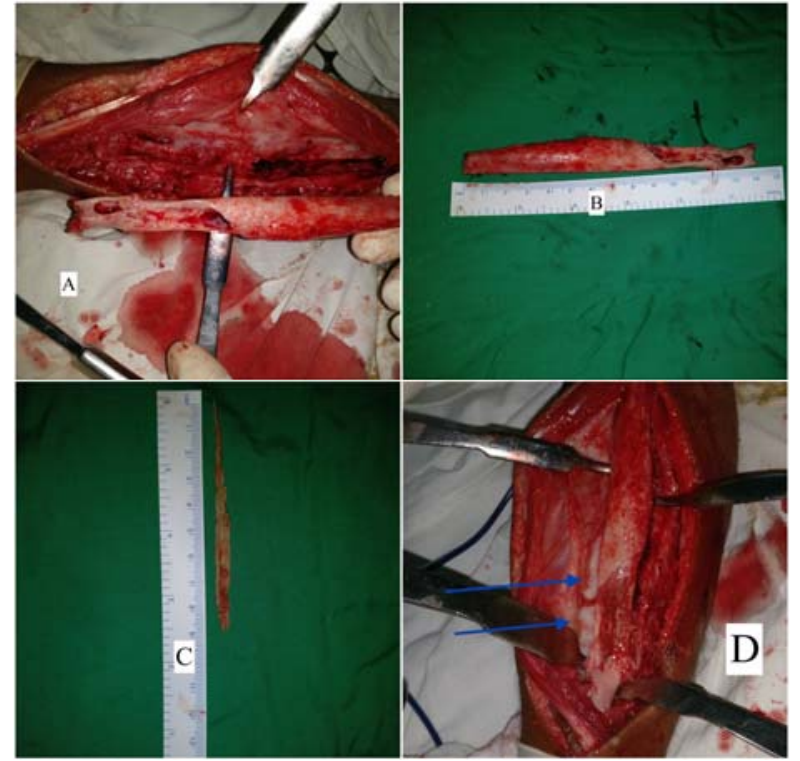

Fig 4 A: Resected Fibular Diaphysis with preservation of periosteum B: Resected Diaphysis C: Sequestrum D: Poor quality and fibrotic periosteum in the distal part (blue arrow). This if found intraoperatively suggest it will form a poor regenerate.

Though we had planned for sequestrectomy, we realized intraoperatively the entire bone was involved hence a resection was done $(14 \mathrm{~cm})$ keeping the periosteum intact ${ }^{[10]}$ (Fig 4). After removal of the sample, patient was given a dose of Cefuroxime $750 \mathrm{mg}$ IV. Distal remnant of fibula $(4 \mathrm{~cm})$ was fixed to the tibia with a syndesmotic screw under C-arm guidance. Intraoperatively the proximal fragment was found to be very mobile. To prevent subsequent knee instability we decided to do a syndesmotic fixation of the proximal fibula remnant to tibia ${ }^{[11]}$. The periosteum was closed with Vicryl No 0 and the remaining wound was closed in layers over a drain. The material obtained were sent for Histopathology and (Aerobic, anaerobic, tubercular and fungal) culture.

Postoperative (Fig 5):

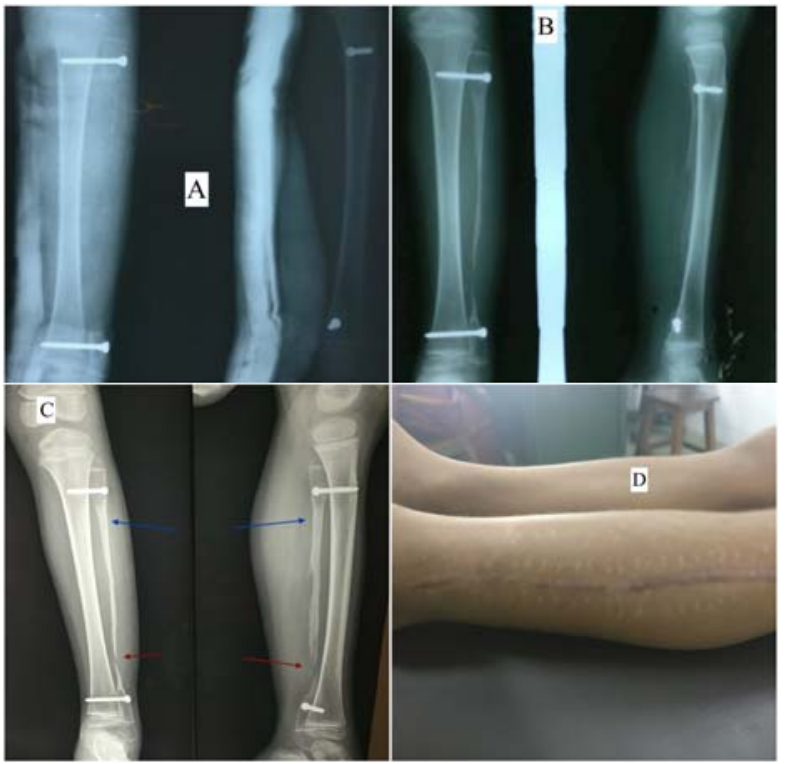

Fig 5 A: Immediate Post-op Xray with the proximal and distal fibula remnant fixed with syndesmotic screw. B: 3 month follow-up Xray

C: 1 year follow-up X-ray with good quality new bone in the proximal and middle segment (blue arrow) but poor quality bone at the site of previous cloacae (red arrow) D: Suture site healed 
Patient was started on IV Linezolid 300mg BD and kept in a slab for one week. The drain was removed on third day. The culture came out to be Methicillin resistant Staphylococcus aureus sensitive to Linezolid. Patient was started on IV 300 $\mathrm{mg}$ Linezolid BD for 3 weeks and oral $300 \mathrm{mg}$ Linezolid BD for three weeks. WBC counts, ESR and CRP were monitored weekly and showed a falling trend. Patient was mobilized three weeks postop and he started running and squatting 6 weeks postop.

Three months follow-up: Heel Valgus of $5^{\circ}$ on the affected side (Fig 6 B). Pain score noted at three months follow-up was Face 2.

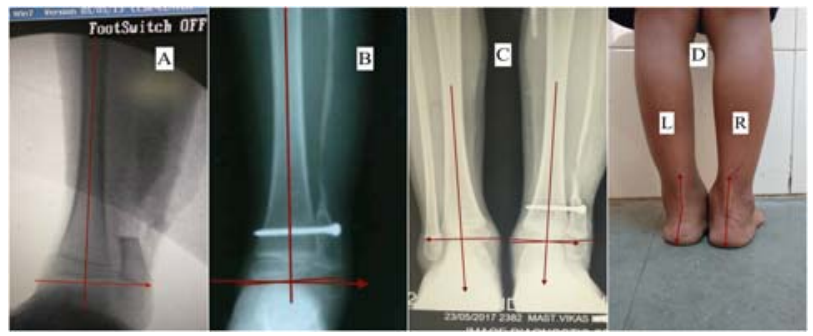

Fig 6: A: Intra-op after fibulectomy B: 3 months post op C: 1 year post-op D: $5^{\circ}$ ankle Valgus in the operated leg (Left) at 1 year Follow-up

Final Follow-up at one year: Heel Valgus of $5^{\circ}$ on the affected side (Fig 6 C,D), Ankle ROM is from 20 degree dorsiflexion to 40 degree plantar flexion on both the sides and pain free, pain score is Face 0 . Patient is able to do all routine activities.

\section{Discussion}

Management of fibular osteomyelitis presents various challenges with respect to stability of ankle mortise, poor quality of periosteum and fibular pseudoarthrosis. Trauma can reactivate a latent infection ${ }^{[12]}$. Progressive valgus deformity at the ankle is a known complication that can occur many years after index fibular resection ${ }^{3}$. Ankle valgus leads to difficulty wearing shoes, inability to walk and mechanical pain ${ }^{[13]}$. Hence whenever possible the continuity of fibula should be maintained. Sequestrectomy should be the initial management with diaphyseal resection reserved for recurrence ${ }^{[1]}$. Diaphyseal resection is necessary in presence of extensive involvement. When resection is done, the distal syndesmotic screw will stabilize the distal fibular remnant (facilitating the weight bearing function) and prevent ankle valgus deformity ${ }^{[6]}$. According to a systematic review done by Oded et al ${ }^{[11]}, 3.9 \%$ of patients with proximal fibula resection had symptomatic knee instability. The proximal segment of fibula was found to be freely moving intra-operatively and hence was stabilized with a proximal syndesmotic screw. Sub periosteal dissection is a prerequisite for regeneration of new bone ${ }^{[11]}$. The quality of the periosteum (an idea of the quality can be obtained by the amount of involucrum formed ${ }^{[14]}$ ) decides the amount of regenerate. In chronic osteomyelitis, in the area of the cloacae the periosteum is of poor quality resulting in poor bone formation. As in our case the new bone formation is abundant in proximal and middle fibula but poor in the distal fibula (Fig 5). Apart from this further anatomical constraints (blood supply of fibula) restrict the regeneration of the fibula especially at the ends. The periosteum of the lower third and majority of the upper third of fibula relies more on the nutrient artery than on adjacent muscle arteries for its blood supply. In contrast, the middle third of the fibula has abundant muscle-periosteal anastomoses. With disruption of the nutrient artery through removal of a fibular segment, the main source of blood supply to the periosteum is disrupted, and ischemia of the periosteum can lead to failure of regeneration of the fibular defect ${ }^{[15]}$. The possibility of fibular pseudoarthrosis must always be kept in mind and reconstructive methods must be used to prevent valgus deformity ${ }^{[16]}$. There are conflicting data in literature regarding the prevention of ankle valgus using a syndesmotic screw. Fragniere et al ${ }^{[9]}$ in their series of 20 cases concluded that valgus deformity of the ankle can be prevented efficiently with a syndesmotic screw. However their study was done in patients with vascularized fibular graft harvesting and had a follow-up of 4.1 years. Soo et al ${ }^{[7]}$ and MS Moon et al ${ }^{[8]}$ concluded supramalleolar synostosis (Lagenskiold operation) to prevent valgus ankle deformities are effective initially however it does not guarantee prevention of ankle valgus. Soo et al. ${ }^{[7]}$ in their series found two $(40 \%)$ out five cases treated with Langenskiold's operation at the index procedure and 10 $(71 \%)$ out 14 cases (initially observation) required supramalleolar corrective osteotomy because of the gradual deterioration of the ankle joint deformities. All of the patients ere followed-up till skeletal maturity. Out of the five cases treated with Langenskiold's operation two were cases of fibular diaphyseal resection done for chronic osteomyelitis of fibula. The cause of valgus deformity even after syndesmotic fixation was thought to be due to the loss of normal physiological loading of distal physis. However the sample size was small and hence a study with larger sample size is needed to validate their claims. These children should be kept in close follow-up till skeletal maturity. At follow-up visit clinical examination and weight bearing mortise and lateral radiographs of ankle should be evaluated for valgus deformity. The radiographic parameters to be monitored are ${ }^{[7,13]}$.

1. Tibio-talar angle (upto $10^{\circ}$ valgus is tolerated)

2. Upward migration of lateral malleolar physis (lateral malleolar physis normally lies below the tibial physis).

3. Reduced growth of the lateral tibial physis.

On retrospectively analyzing the X-ray, we found that perhaps the $5^{\circ}$ valgus could have occurred intra-operatively while tightening the syndesmotic screw. We inserted the syndesmotic screw after resection of fibula, this could have resulted in a valgus force at the ankle leading to a valgus deformity (Fig 6) (Fig 7).

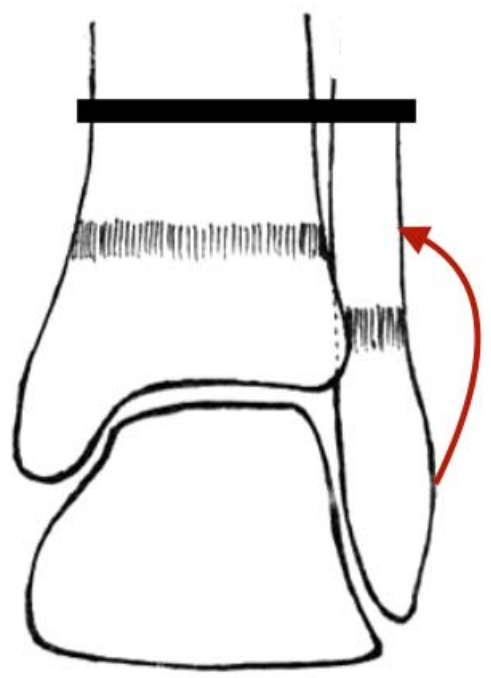

Fig 7: Valgus effect of syndesmotic screw when inserted after fibulectomy. 
This could have been avoided by inserting the syndesmotic screw before fibular resection. This cause should also be kept in mind while evaluating such patients. At the recognition of progressive valgus deformity prophylactic surgery in the form of distal medial tibia hemiepiphysiodesis with either 8 plate or cannulated screws or should be carried out ${ }^{[17]}$.

Take home message

1. Sequestrectomy should be preferred to diaphyseal resection to prevent loss of fibular continuity.

2. Syndesmotic fixation of distal fibular remnants will stabilize the ankle

3. Syndesmotic fixation should be performed before fibular resection.

4. Such patients should be followed up till skeletal maturity.

\section{References}

1. Ziani FM. The clinical result of 20 cases of chronic haematogenous osteomyelitis of the fibula treated by operation. Journal of pediatric Orthopaedics. 1991; 11(1):138.

2. Paluska DJ, BW. Ankle Valgus after Grice Subtalar Stabilization: The Late evaluation of personal series with a modified technique. Clin Orthop Relat Research, 1968; 59:137-46.

3. Wiltse LL. Valgus deformity of the ankle: A sequel to accquired or congenital abnormalities of fibula. J Bone Joint Surg (Am). 1972; 54A:595-606.

4. Babhulkar SS, KC. Ankle instability after fibular resection. J Bone and Joint Surgery (Br). 1995; 77:25861.

5. Langenskiold A. Pseudarthrosis of the fibula and progressive valgus deformity of the ankle in children: treatment by fusion of the distal tibial and fibular metaphyses: review of three cases. J Bone Joint Surg Am. 1967; 49(3):463-470

6. Goh JC MA. Biomechanical study on the load bearing characteristics of fibula and the effects of fibular resection. Clinical Orthopaedics \& Related Research. 1992; 279:223-8.

7. Soo Hwan Kang MS. Ankle Deformity Secondary to Acquired Fibular Segmental Defect in Children. Clinicsin Orthopedic Surgery. 2010; 2:179-185.

8. MS Moon RS. Valgus ankle secondary to acquired fibular pseudoarthrosis in children. Long-term results of the Langenskiöld operation. M S Moon; S K Rhee; H D Lee; I T Ju; S H Nam. Bulletin (Hospital for Joint Diseases (New York, N.Y.); 1997; 56.

9. Fragnière B, WP. Prevention of ankle valgus after vascularized fibular grafts in children. Clin Orthop Relat Res. 2003; 408:245-51.

10. Mohammed AlMaiyah, MV. Recurrence of Chronic Osteomyelitis in a Regenerated Fibula After 65 Years. Orthopedics, 2007; 30(5):403-404.

11. Oded Ben Amotz MD, RR. Complications related to harvest of the proximal end of the fibula: A systematic review. Microsurgery, 2014; 34(8):666-669.

12. Law MD, SR. Late infection in healed fractures after open reduction and internal fixation. Orthop Rev, 1993; 545-52.

13. Aurégan JC, GF. Children ankle valgus deformity treatment using a transphyseal medial malleolar screw. Orthopaedics \& Traumatology: Surgery \& Research, 2011; 97:406-409.

14. Herring JA. Tachdijan's Pediatric Orthopaedics (5th ed., Vol. 2). Elsevier.
15. Hsu LC, YA. Valgus deformity of the ankle resulting from fibular resection for a graft in subtalar fusion in children. 1972; 54:585-594.

16. González-Herranz PD, RM. Valgus deformity after fibular resection in children. J Pediatr Orthop. 2003; 23(1):55-59.

17. Peter M, Stevens JM. Guided Growth for Ankle Valgus. J Pediatr Orthop. 2011; 31(8):878-883. 